\title{
PULMONARY ATRESIA WITH INTACT VENTRICULAR SEPTUM*
}

BY

\author{
LARRY P. ELLIOTT, PAUL ADAMS, JR., AND JESSE E. EDWARDS \\ From the Departments of Pediatrics and Pathology, University of Minnesota, Minneapolis, and the Department of \\ Pathology, The Charles T. Miller Hospital, St. Paul, Minnesota, U.S.A.
}

Received November 30, 1962

Although congenital pulmonary atresia with an intact ventricular septum is an uncommon malformation, its recognition is important because the results of surgical treatment may be favourable in some cases. Functionally, this entity belongs to a group of congenital malformations each having obstruction to pulmonary flow. This group includes severe pulmonary stenosis, tricuspid atresia, pulmonary atresia with a ventricular septal defect, and the origin of the aorta and pulmonary trunk from the right ventricle with severe pulmonary stenosis.

In these congenital cardiac malformations, the fundamental functional problem and the clinical picture are determined by obstruction to pulmonary blood flow in the right side of the heart. As a result of this obstruction, right-to-left shunts of varying degrees occur at different sites depending on the type of intracardiac communication present. This hæmodynamic phenomenon is usually manifested clinically by early intense cyanosis. Congestive heart failure occurs in most cases, and early death is common. Since the results of surgical treatment vary greatly in cases of these types of cardiac malformation, immediate and proper diagnosis is essential (Benton et al., 1962).

Greenwold et al. (1956) made a study of 12 cases of pulmonary atresia with an intact ventricular septum and divided them into two groups on the basis of the size of the right ventricle. The first group included 6 cases in which the right ventricle was minute or small, and the second group included 6 in which it was of normal size or large. Keith, Rowe, and Vlad (1958) described the pathological findings in 46 cases: the right ventricle was of normal size or large in only 15 per cent of the 46 cases. Davignon et al. (1961) correlated the clinical and pathological findings in 20 cases: several of these were previously included in the report by Greenwold et al. (1956).

\section{MATERIAL}

This report is based on the anatomical, clinical, and electrocardiographic aspects of 12 cases of pulmonary atresia with an anatomical or functionally intact ventricular septum, which were observed at the University of Minnesota Hospitals. In an article to be published, Kieffer and Carey (1963) will consider the radiographic aspects of these cases. Other examples of obstructive lesions of the right side of the heart, such as tricuspid atresia, were not included in our study.

\section{ANATOMICAL OBSERVATIONS}

The right ventricle was minute in 3 cases, small in 6 , of normal size in 2 , and large in 1 case.

Pulmonary Valve and Arteries. The pulmonary valve had no opening but was represented by a fibrous membrane or diaphragm (Fig. 1). Radiating from the centre on the arterial side of the diaphragm were three equidistant ridges or raphes which extended to the wall of the pulmonary trunk.

* This study was supported by Research grant HE-5694 from the National Heart Institute, United States Public Health Service. 
The diameter of the aortic trunk was $1 \cdot 2$ to 6 times as great as the diameter of the pulmonary trunk. The 12 cases were divided into three groups on the basis of the relation of the diameter of the pulmonary trunk to that of its two main branches. The first group included 6 cases in which

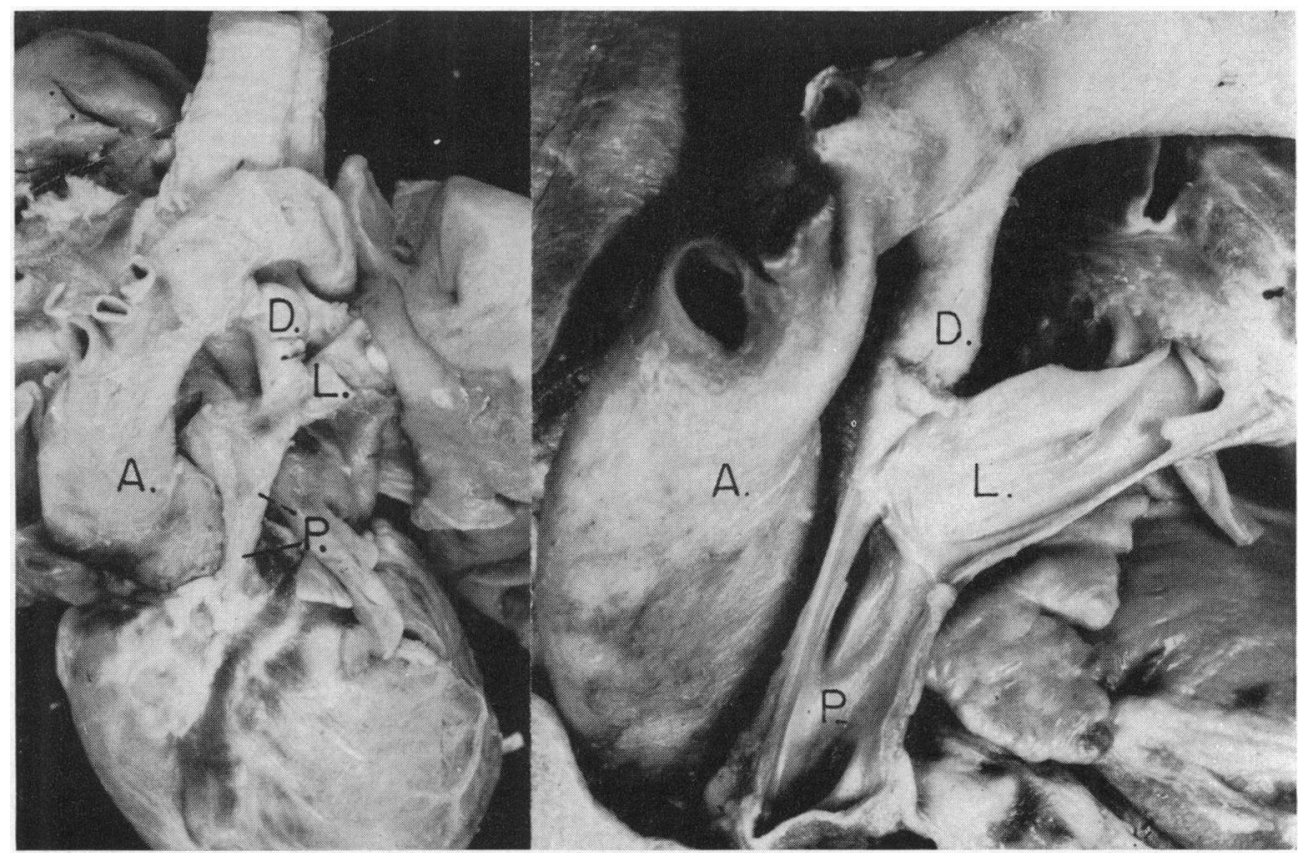

FIG. 2.-Two of three types of pulmonary arterial trunk observed in cases of pulmonary atresia with an intact ventricular septum. Left. The pulmonary trunk $(\mathrm{P})$ is narrow and divides into the larger right and the left (L) pulmonary artery. $A=$ ascending aorta; $D=$ ductus arteriosus. Right. The pulmonary trunk (P), and the left main branch (L) which is relatively wide. Abbreviations as in left. 


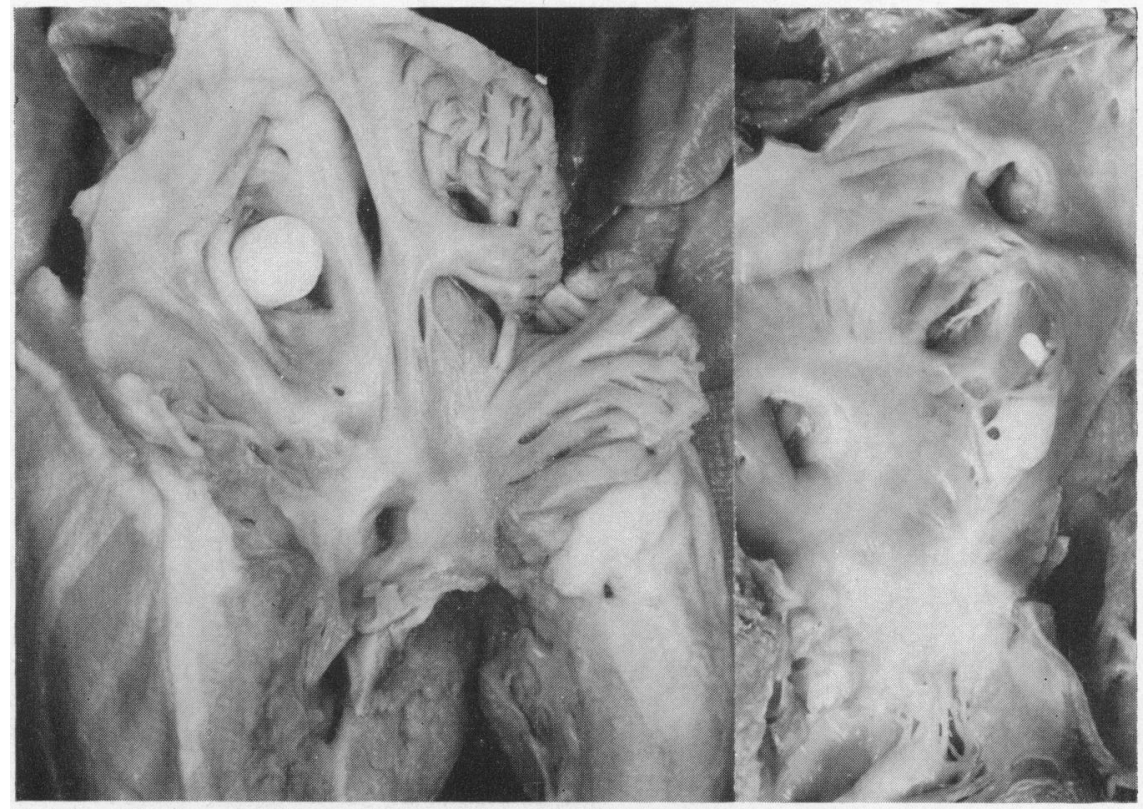

FIG. 3.-Interatrial communications in two cases. Left. A small defect in the atrial septum (containing probe) of the fossa ovalis type viewed from the right side. Right. Left atrium. An aneurysm of the fossa ovalis projects into the left atrium below the probe. This is anatomical evidence that the pressure in the right atrium was higher than that in the left atrium.

the right and left branches were of the same diameter as the parent vessel which was narrow and hypoplastic. The second group included 4 cases in which the right and left branches were about twice as wide as the pulmonary trunk which was small and hypoplastic (Fig. 2, left). The third group included 2 cases in which the diameter of the two branches was the same as that of the pulmonary trunk which was rather wide (Fig. 2, right). In each case, the aortic arch coursed over the left main stem bronchus as a left aortic arch (Fig. 2, left).

The relation of the ascending aorta and the pulmonary trunk was the same as that observed in normal infants. The positions of the pulmonary and aortic valves likewise were the same as that observed normally.

Communication Between the Lesser and Greater Circulations. A patent ductus arteriosus was present in 11 cases (Fig. 2). The internal diameter of the ductus arteriosus ranged from 1 to $6 \mathrm{~mm}$. and averaged $2.5 \mathrm{~mm}$. In 2 cases, the ductus was unusually long and measured 8 and $9 \mathrm{~mm}$. respectively.

An interatrial communication was present in all of the 12 cases. A valvular-competent foramen ovale was present in 5 and an atrial septal defect of the fossa ovalis was present in 7 (Fig. 3, left). The diameter of the defect ranged from 4 to $6 \mathrm{~mm}$. In 2 of the 5 cases in which there was a valvular-competent foramen ovale, an aneurysm of the fossa ovalis directed to the left also was observed (Fig. 3, right). This is indirect evidence of extreme pressure on the right side of the heart.

A communication between a coronary artery and the right ventricle, as described by Williams, Kent, and Edwards (1951), was present in 4 cases.

In 4 of the 9 cases in which the right ventricle was minute or small and there was evidence that the tricuspid valve was functionally competent, enlarged sinusoids proximal to the pulmonary valve 
penetrated the right ventricular myocardium and converged near the epicardium (Fig. 4, left). At the point of convergence, these sinusoids formed a single vessel which entered the epicardium and communicated with the anterior descending coronary artery at the inferior aspect of the heart (Fig. 4, left) near its junction with the posterior descending coronary artery. The coronary artery that participated in this communication was dilated and tortuous (Fig. 4, right). At the site where this anomalous vessel emerged from the myocardium, there was a dimple.

A small defect of the ventricular septum, which was $1 \mathrm{~mm}$. in diameter, was observed in one case (Fig. 5). When the defect was viewed from the right side, it was situated above the septal leaflet of the tricuspid valve and the leaflet was inserted below the annulus of the ring of the tricuspid valve (Fig. 5, left). This allowed the defect to lie below the valve annulus and yet above valvular tissue (Fig. 5, centre). In addition, there was a polyp-like collection of loose fibrous tissue adherent to the postero-inferior rim of the defect. This tissue and the septal leaflet of the tricuspid valve appeared to have had the ability to seal this defect like a shutter during closure of the valve during systole.

When viewed from the side of the left ventricle (Fig. 5, right), the centre of the defect was below the junction of the right and posterior aortic cusps and was separated from these structures by a thin $0.5 \mathrm{~mm}$. rim of muscular tissue.

Although this case cannot be classified as a case of pulmonary atresia with an intact ventricular septum in the strictest anatomical sense, the size of the defect, the shutter-like mechanism of the tricuspid valve, and the presence of other anatomical features of pulmonary atresia with an intact ventricular septum indicate a functional and nearly anatomical identity between this case and the others in this study. If realistic clinico-pathological correlations are to be observed and recorded, it is important that minor variants such as this be included. 


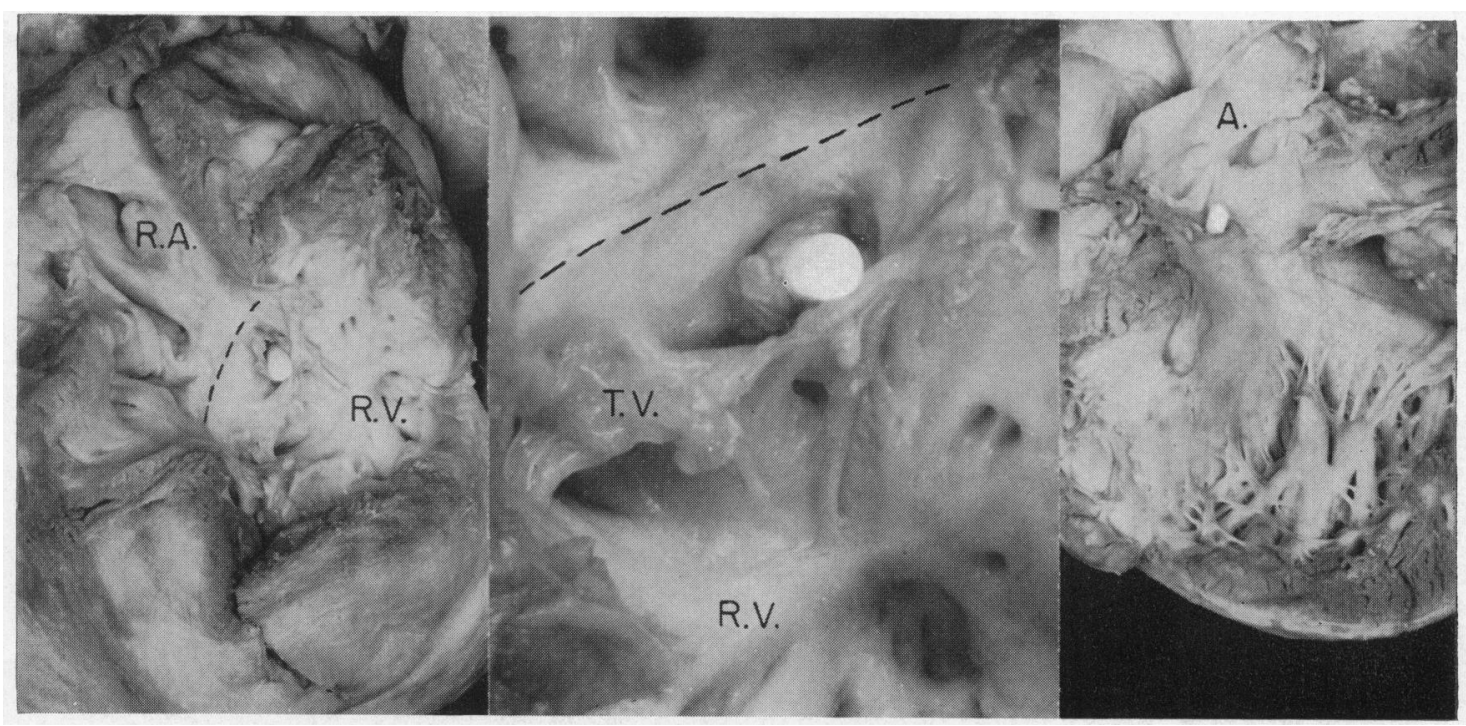

FIG. 5.-Ebstein-like malformation of the tricuspid valve and a small defect in the ventricular septum leading to a communication between the left ventricle and the right atrium. Left. Interior of right atrium (RA) and right ventricle (RV). The probe lies above the tricuspid valve and leads to the communication between the left ventricle and that part of the right ventricle which lies above the tricuspid valve. The tricuspid valve is displaced downward from the annulus fibrosus (dotted line) representing the Ebstein type of malformation. The right ventricle (RV) is comparatively small and the endocardium is thick. Centre. Posterior view of the tricuspid valve (TV) and adjacent structure. The dotted line indicates the level of the annulus fibrosus of the tricuspid valve that has been displaced downward in a typical Ebstein-like deformity. $R V=$ right ventricle. The probe lies in the small defect in the ventricular septum, which, because of the associated malformation of the tricuspid valve, causes the communication to lie between the left ventricle and the "atrial" portion of the right ventricle. Right. Outflow portion of the left ventricle. The probe lies in the ventricular septal defect which is centred below the commissure that joins the right and posterior cusps of the aorta (A).

The Tricuspid Valve. The functional explanation for the two types of right ventricle described by Greenwold et al. (1956) and later emphasized by Davignon et al. (1961) is primarily based on the concept of a competent tricuspid valve in cases in which the right ventricle is small or minute and an incompetent tricuspid valve in cases in which the right ventricle is of normal size or large.

In the 2 cases in which the right ventricle was of normal size, the septal leaflet of the tricuspid valve was displaced below the annulus fibrosus in a manner similar to that observed in cases of Ebstein's malformation of the tricuspid valve (Fig. 6, upper left). In the one case in which the right ventricle was large, the tricuspid valve was inserted at the annulus fibrosus in the usual manner but was deformed, i.e. the valvular tissue was redundant, gnarled, and fused to large, thick papillary muscles without chordal attachments (Fig. 6, upper right). In addition, the edges of the valve were thick and rolled. In the 2 cases in which the right ventricle was of normal size and in the 1 case in which it was large, the deformities appeared to render the valve incompetent.

In the 9 cases in which the right ventricle was minute or small, there was a relation between the size of the orifice of the tricuspid valve and the size of the ventricle, and the chordal attachments were normal. In 7 of these 9, the most striking observation was an Ebstein-like malformation that involved only the septal leaflet of the tricuspid valve or also involved part of the posterior leaflet (Fig. 6, lower left and lower right). In each of these 7 cases, the septal leaflet was inserted 2 to $4 \mathrm{~mm}$. below the annulus (Fig. 7). As far as we have been able to determine, an Ebstein-like deformity of 
FIG. 6.-Variations in Ebstein-like malformation and in size of the right ventricle in four cases. Upper left. Right ventricle (RV) is of normal size. Dotted line indicates level of annulus fibrosus of the tricuspid valve. The adjacent portions of the septal and posterior leaflets are displaced downward and attached to the wall of the right ventricle rather than at the level of the annulus fibrosus. Upper Right. The right ventricle is large, and the right atrium (RA) is of giant proportions. Dotted line indicates the level of the annulus fibrosus. Although the base of the tricuspid valve is attached almost entirely to the annulus fibrosus, adhesions of the posterior and septal leaflets of the tricuspid valve to the wall of the right ventricle are similar to those that may be seen in cases of well-established malformation of the tricuspid valve. In addition to these changes, the valvular tissue, especially that of the anterior leaflet (right side of illustration) is spongy. A thick column of muscle (B) extends from the ventricular septum to the anterior wall of the right ventricle. This may represent an enlarged moderator band. Lower left. The small right ventricle (RV) and the basal attachment of the septal and posterior leaflets of the tricuspid valve lie below the level of the annulus fibrosus (dotted line). In addition to these changes, the free edge of the involved portion of the valve is thickened. Lower right. Adjacent portions of the septal leaflet (SL) and the posterior leaflet (PL) of the tricuspid valve are attached to the wall of the right ventricle below the level of the annulus fibrosus (dotted line). The right ventricle is small. Characteristically, the endocardium of this type of ventricle is thickened. 


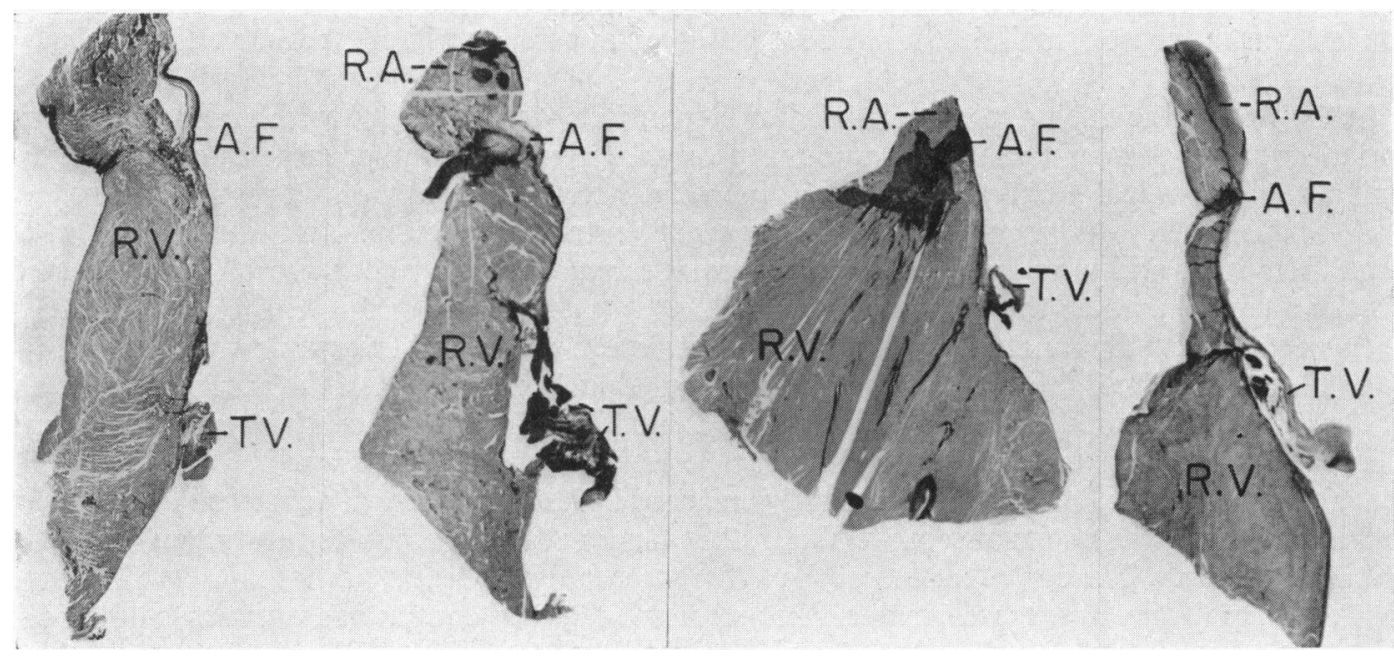

FIG. 7.-Low-power photomicrographs of sections taken through the right atrium, right ventricle, and septal and posterior leaflets of the tricuspid valve in 4 cases. $A F=$ annulus fibrosus; $R A=$ wall of right atrium; $\mathrm{RV}=$ wall of right ventricle; $\mathrm{TV}=$ tricuspid valve. Left. Right ventricle is large and the tissue of the tricuspid valve is attached to the wall of the right ventricle at several places. Left centre. Base of tricuspid valve is attached to the wall of the right ventricle well below the annulus fibrosus. The right ventricle is small. Right centre. Base of tricuspid valve is attached to the wall of the right ventricle. The right ventricle is small. Right. The base of the tricuspid valve is attached to the wall of the right ventricle in at least two places. The right ventricle is small and its basal aspect is atrophic.

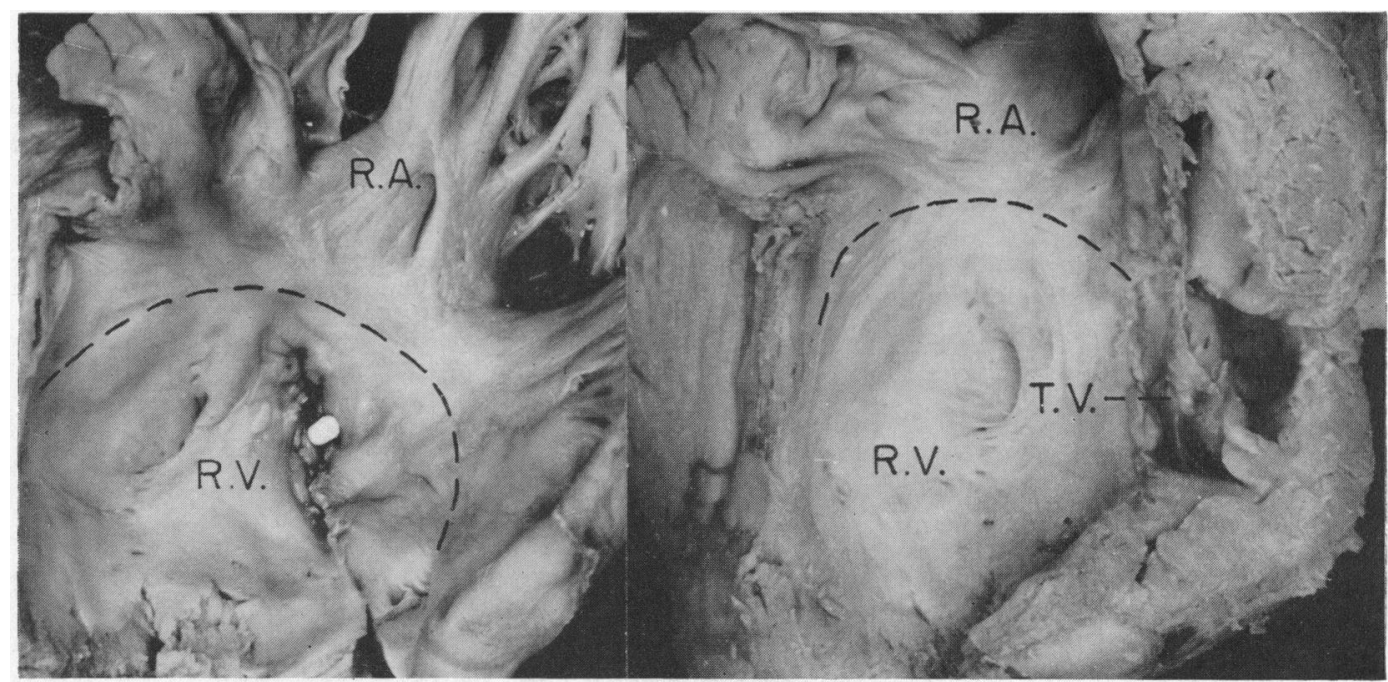

FIG. 8.-Left. Right atrium (RA) and that portion of the right ventricle (RV) which lies above the level of the tricuspid valve (containing probe). Because of pronounced downward displacement of the tricuspid valve, a consider able portion of the inflow portion of the right ventricle joins with the right atrium to form a common receiving chamber. Level of the annulus fibrosus is indicated by the dotted line. Right. Posterior aspect of the right ventricle (RV) and right atrium (RA). Level of the annulus fibrosus is indicated by the dotted line. The tricuspid valve (TV) is displaced downward so that no valvular tissue appears along the posterior aspect of the right ventricle (RV) except at the apical portion, which lies toward the right of the illustration. RA=right atrium. 
the tricuspid valve has not been described previously in cases of pulmonary atresia in which the right ventricle was minute or small, but it has been observed in cases in which the ventricle was of normal size or large.*

In the 7 cases in which a minute or small right ventricle was associated with an Ebstein-like malformation of the tricuspid valve, the amount of valvular tissue was rather large as compared to the small size of the valve. This suggested that the valve had functioned without regurgitation.

Right Ventricle. As previously stated, the right ventricle was minute in 3 cases, small in 6, of normal size in 2, and large in 1 case. Endocardial sclerosis of the right ventricle was observed in 2 of the 6 cases in which the ventricle was small.

Right Atrium. The anatomical relation and structure of the right ventricle were normal in all of the 12 cases. The right atrium was slightly enlarged in 1 of the 3 in which the right ventricle was minute, and it was moderately enlarged in the 2 remaining cases. The right atrium was much enlarged in the 3 cases in which the right ventricle was of normal size or large.

Left Atrium. The left atrium was slightly enlarged in 9, and moderately enlarged in 3 cases. In the 3 latter, there was an atrial septal defect rather than a valvular-competent foramen ovale.

Left Ventricle. The basic features of the left ventricle were the same as those observed in normal hearts. The mitral valve was normal and its anterior leaflet was continuous with tissue of the aortic valve. The left ventricle was hypertrophied and dilated in each case. This was indirect anatomical evidence of an increased flow of blood through the left side of the heart.

Venous Connexions. The connexions of the systemic, coronary, and pulmonary veins were normal.

\section{ElECTROCARDIOGRAPHIC OBSERVATIONS}

In pulmonary atresia with an intact ventricular septum, the electrocardiogram may show characteristic differences between cases in which the right ventricle is minute or small and those in which it is of normal size or large (Benton et al., 1962; Davignon et al., 1961; Keith et al., 1958).

$P-R$ Interval and $P$ Waves. In all of the 12 cases, the duration of the $P-R$ interval was normal for the age of the patient and the rate of the heart. The average mean $P$ vector in the frontal plane was +60 degrees. The right atrium was enlarged in 4 of the 9 cases in which the right ventricle was minute or small (Fig. 9, lower left). The left atrium was enlarged in 2 cases (Fig. 9, lower right), both atria were enlarged in 2 , and the contour of the $P$ wave was normal in the remaining case. In one case enlargement of the right atrium was observed when the patient was 1 day old (Fig. 10, lower left). In 2 cases, cardiographic evidence of enlargement of the right atrium appeared initially in lead V2 as tall peaked $P$ waves without alteration of the $P$ waves in any other lead.

The right atrium was enlarged in 1 of the 3 cases in which the right ventricle was of normal size or large, and both atria were enlarged in the 2 remaining cases.

Mean QRS Axis and QRS Spatial Vector Observations in the Frontal and Horizontal Planes. In the 9 cases in which the right ventricle was minute or small, the mean manifest electrical axis of the QRS loop varied between +150 degrees (right axis deviation) and -105 degrees (left axis deviation). Despite this wide variance, the QRS axis in the frontal plane was normal in 7 of the 9 cases and was confined between +95 degrees and +65 degrees. In all of the 9 cases, the duration of the QRS interval was $0.05 \mathrm{sec}$. or less.

In 2 cases in which the right ventricle was of normal size or large, the mean manifest electrical axis in the frontal plane was normal: it was +90 degrees in one and +60 degrees in another. In the

\footnotetext{
* In a case of pulmonary atresia with intact ventricular septum that was observed after the completion of this study, there was a fully-developed Ebstein malformation of the tricuspid valve. The posterior and septal leaflets of the valve were attached to the apical wall of the right ventricle (Fig. 8). The base of the anterior leaflet of the valve was attached to the annulus. There was considerable endocardial thickening of the wall of the right ventricle proximal to the tricuspid valve. Distal to the valve the right ventricle was small and its wall was hypertrophied.
} 


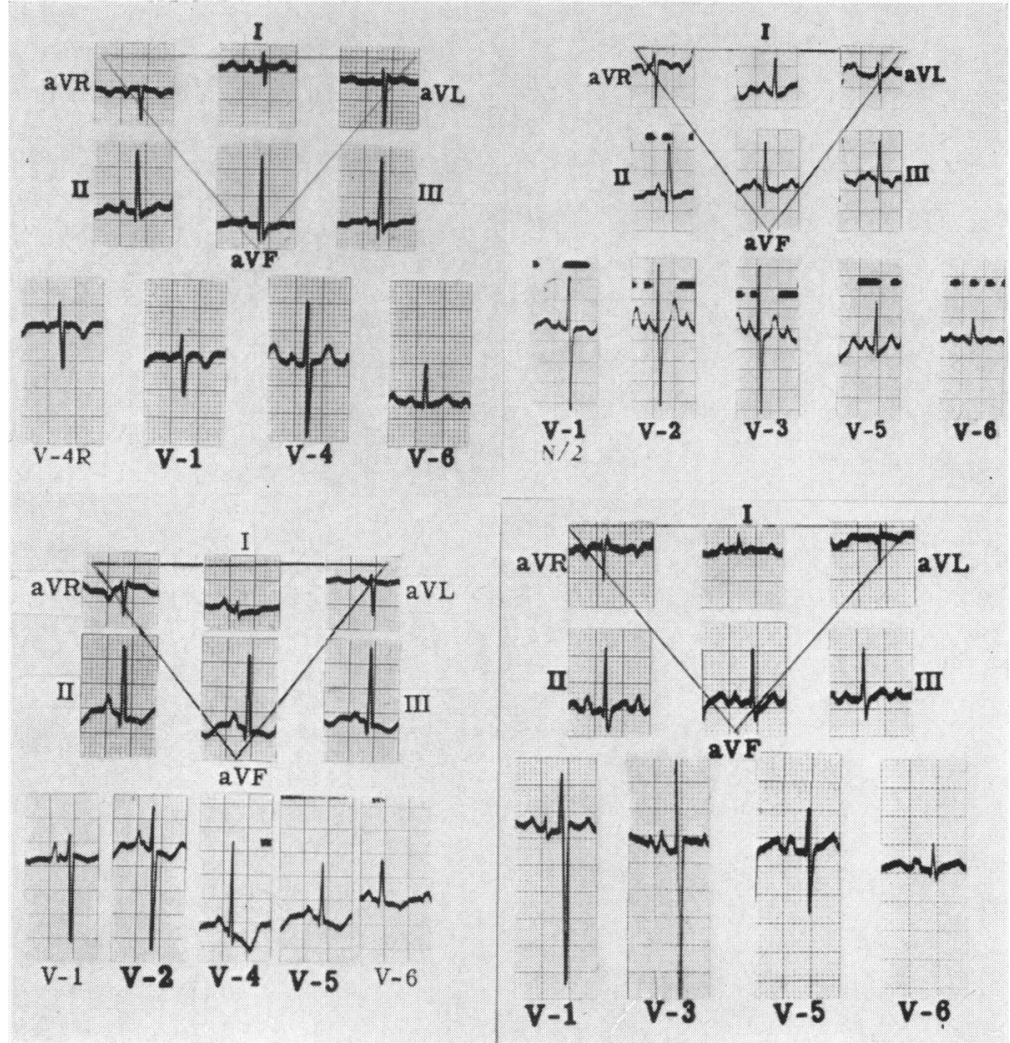

FIG. 9.-Electrocardiograms obtained in four cases of pulmonary atresia in which the right ventricle was minute or small. Each tracing suggests hypertrophy of the left ventricle. Upper left. From a 4-day-old infant. Lower left. From a 4-week-old infant. Upper right. $\mathrm{R}$ wave of more than $20 \mathrm{~mm}$. suggests additional hypertrophy of the right ventricle. Lower right. From a 1-week-old infant.

remaining case in which the right ventricle was of normal size or large, the mean manifest electrical axis in the frontal plane was of the right axis deviation type (+135 degrees).

In 7 of the 9 cases in which the right ventricle was minute or small, the QRS loop in the horizontal plane was counterclockwise and situated predominantly posteriorly and to the left, being represented by an rS pattern in lead V1 and a qR, qRs, or RS pattern in lead V6 (Fig. 9). This situation of the QRS loop and its representative cardiographic pattern were consistent with left ventricular hypertrophy in these 7 cases. The relatively deep S wave observed in lead V6 in 2 of these cases was the extension of the terminal QRS loop in a right posterior location beyond the iso-electric line of lead V6 (Fig. 9, lower right) and should not be interpreted as an additional sign of right ventricular hypertrophy.

Keith et al. (1958) occasionally used the absence of a relatively deep S wave in lead V6 in their cases of tricuspid atresia to distinguish these from cases of pulmonary atresia. In our experience, this finding has not been reliable in distinguishing tricuspid atresia from pulmonary atresia.

In the remaining 2 cases in which the right ventricle was small or minute, cardiography disclosed hypertrophy of the right ventricle (Fig. 10), which was an uncommon finding in cases previously reported. In these two, the QRS vector loop in the horizontal plane was essentially clockwise 


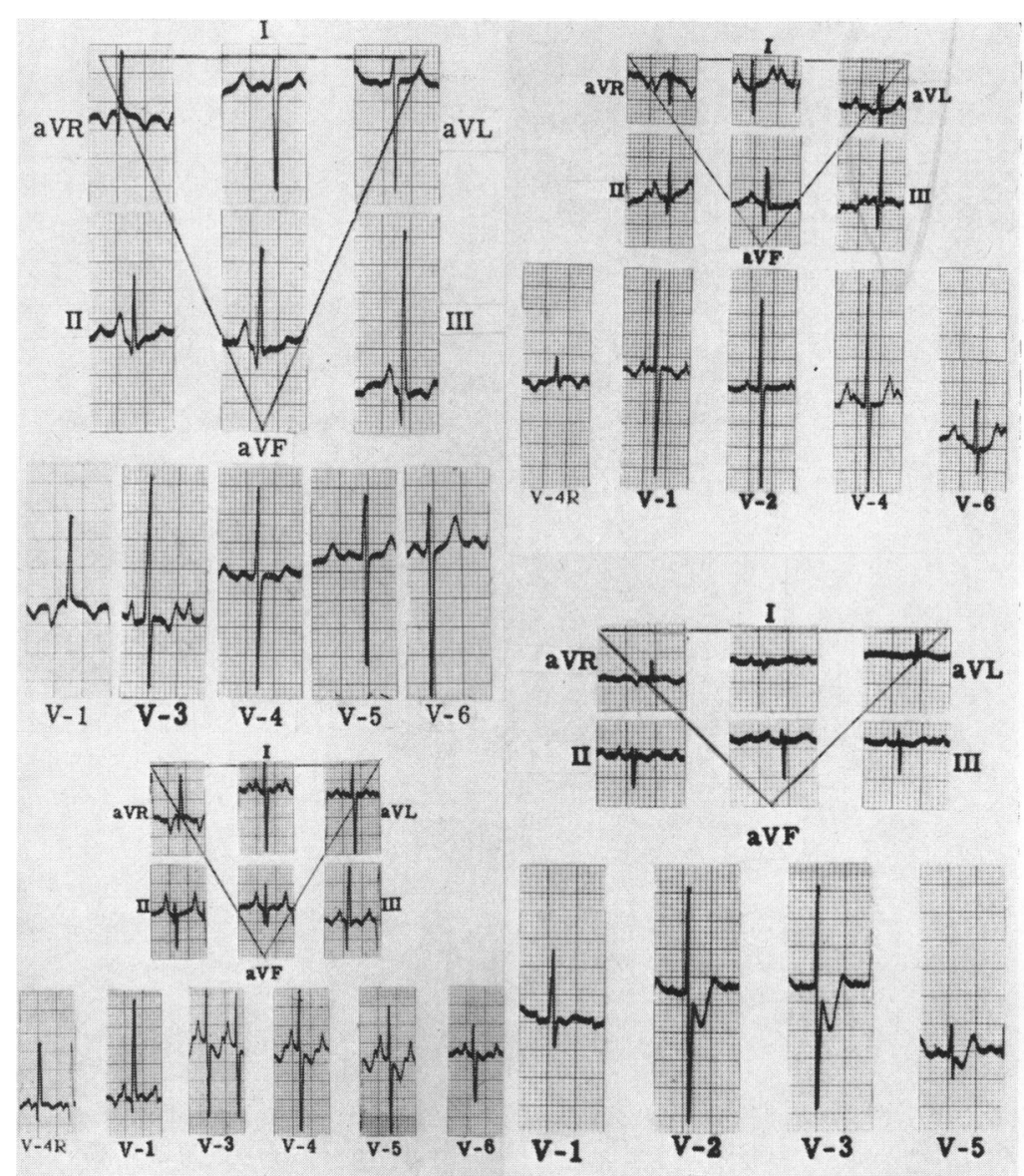

FIG. 10.-Electrocardiograms made in four cases in which the right ventricles were of various sizes. Upper left. From a 1-month-old infant. The right ventricle was large. The $\mathrm{qR}$ pattern in lead V1 is evidence of hypertrophy of the right ventricle. Upper right. Showing evidence of hypertrophy of both ventricles in a 3-month-old infant. The right ventricle was large. Lower left. Showing evidence of hypertrophy of the right ventricle in a 6-day-old infant. The right ventricle was small. Lower right. Showing evidence of hypertrophy of the right ventricle in a 2-week-old infant. The right ventricle was small.

and situated predominantly anteriorly and to the right. This situation yielded an Rs pattern in lead V1 in one, and it yielded an rsR' pattern in the other. The R/S ratio in lead V1 was above one in these 2 , and less than one in the aforementioned 7 cases.

In analysing the horizontal plane (præcordial leads) of the QRS spatial vector in the 3 cases in which the ventricle was of normal size or large, it was found that the initial QRS components generated from the right ventricle were directed more anteriorly than they were in 7 of the 9 in which the ventricle was minute or small. This initial anterior orientation was interpreted as indicating right ventricular hypertrophy in each case observed (Fig. 10). In one, the initial vector was posteriorly directed and produced a $\mathrm{Q}$ wave in lead V1 (Fig. 10, upper left).

$T$ Waves. In 5 of the 9 cases in which the right ventricle was minute or small, the mean $T$ vector in the frontal plane varied between +85 degrees and +15 degrees (normal range). It was -90 
degrees in one of the 9 cases and +150 degrees in another. The $\mathrm{T}$ wave in lead V1 was positive in 4 cases. In 3 of these 4 , the positive $T$ wave in $V 1$ should not be interpreted as an additional sign of right ventricular hypertrophy but merely as the result of a widening of the QRS-T angle, presumably the electrical manifestation of left ventricular strain or ischæmia (Fig. 9, lower right).

In 2 of 3 cases in which the right ventricle was of normal size or large, the mean $\mathrm{T}$ vector in the frontal plane was zero degrees, and directed posteriorly. In the remaining case the mean $\mathrm{T}$ vector in the frontal plane was perpendicular and oriented anteriorly in the horizontal plane (præcordial leads).

Correlation Between Age of Patients and Time of Electrocardiography. In 5 of the 9 cases in which the right ventricle was minute or small, the initial cardiogram was made when the infants were 1 week old or less. In 2 cases, cardiography revealed right ventricular hypertrophy of greater magnitude than that observed normally in infants without cardiac disease. In another case, a cardiogram that was made two weeks after the initial one revealed the development of left ventricular preponderance (Fig. 9, upper right).

In 3 other cases, left ventricular preponderance was observed when the infants were less than 1 week old, and in one of these it was observed as early as 1 day.

Special Cases. Left axis deviation was observed in 2 cases (Fig. 10, lower right). The electrical axis of the QRS loop was -105 degrees in one of these and -75 degrees in the other. The right ventricle was small in the former and large in the latter. This pattern of left axis deviation associated with a counterclockwise inscription of the QRS loop in the frontal plane and right ventricular preponderance resembles the electrodynamic changes observed in cases of defects of the persistent common atrioventricular canal (Burchell, DuShane, and Brandenburg, 1960).

\section{Clinical Observations}

The clinical findings in cases of pulmonary atresia with an intact ventricular septum differ only in degree from those in cases of pulmonary stenosis with an intact ventricular septum (Benton et al., 1962). In cases of pulmonary atresia with an intact ventricular septum, the signs and symptoms are determined chiefly by the length and width of the patent ductus arteriosus. After the ductus undergoes the normal obliterative process, the principal flow of blood to the lungs is stopped and the patient usually dies. Collateral vessels to the lungs seem inadequate to sustain life.

Cyanosis, often of an intense degree, is commonly noted before the infant is 2 days old. The infants are well nourished and well developed. Additional non-cardiac anomalies, including hypertelorism, are rarely, if ever, present.

Concomitant with the onset of cyanosis or shortly thereafter, congestive cardiac failure was observed in each case in our series. In contrast with most congenital malformations producing congestive cardiac failure, dyspnœa was not usually evident until frank congestive failure had developed. Clinically, deterioration was rapid, and death occurred between the ages of 1 day and 3 months.

Clinical examination of the cardiovascular system did not reveal any difference between cases in which the right ventricle was small or minute and cases in which it was of normal size or large. A thrill was not evident in any of our cases, but Davignon et al. (1961) reported that a thrill was obtained in two of theirs: in each of these, the right ventricle was of normal size or large and the tricuspid valve was malformed.

In 7 of our 9 cases in which the right ventricle was minute or small, a systolic murmur grade 2 to 3 (scale of 1 to 6) was heard along the lower part of the left border of the sternum. No murmur was heard in the 2 remaining cases. In 1 of the 3 in which the right ventricle was of normal size or large, a murmur was present at birth but disappeared by the tenth day: in the 2 others, auscultation revealed systolic murmurs similar to those heard in 7 of the 9 cases in which the right ventricle was minute or small. A diastolic murmur was not heard in any of our cases. Continuous murmurs occasionally were heard in the cases observed by Keith et al. (1958), but such murmurs were not heard in any of our cases. 
Although each infant was in a state of congestive heart failure at the time of initial examination, the lungs were clear to auscultation in all but one of our cases. The liver was enlarged in all, but pulsation of this organ was not evident. The peripheral pulse was either normal or of low intensity. The low intensity presumably was a manifestation of congesive heart failure and low cardiac output.

Keith et al. (1958) usually did not observe any cardiac murmurs whereas Davignon et al. (1961) observed a systolic murmur of variable intensity in most of their cases.

\section{COMMENT}

As in cases of pulmonary atresia with an intact ventricular septum reported by Greenwold et al. (1956) and by Davignon et al. (1961), our 12 cases could be divided into two groups depending on the size of the right ventricle. The right ventricle was minute or small in 9 of the 12 , and it was of normal size or large in the 3 remaining cases.

Our anatomical study revealed that malformations of the tricuspid valve, particularly Ebstein's malformation, are commonly associated with atresia of the pulmonary valve. In earlier studies, the association of Ebstein's malformation of the tricuspid valve was considered to be the basis for tricuspid regurgitation, and this in turn was believed to be the basis for the development of the right ventricle to normal size or to a size larger than normal. Not previously noted was the occurrence of Ebstein's malformation of the tricuspid valve in cases in which the right ventricle was small. Some degree of Ebstein's malformation was present in 7 of the 9 cases in which the right ventricle was minute or small. As previously noted, valves that were deformed in this manner appeared to be competent when the right ventricle was minute or small. Indeed, the very fact that the right ventricle is minute or small may depend on the tricuspid valve's functioning without regurgitation even though it may be malformed.

The history and auscultation were of no value in distinguishing cases in which the right ventricle was minute or small from those in which it was of normal size or large. It is important to try to make this distinction since the relief of obstruction of the pulmonary valve may produce acceptable functional results, in spite of coexisting tricuspid insufficiency, in cases in which the right ventricle is of normal size or large. On the other hand, surgical correction of the obstruction of the pulmonary valve probably would not produce any benefit in cases in which the right ventricle is minute or small since the small size of the right ventricle would represent an obstructive factor to the flow of blood into the right side of the heart.

Distinction of cases in which the right ventricle was minute or small from those in which it was of normal size or large appeared to depend largely on the radiographic and electrocardiographic findings. In cases in which the right ventricle was minute or small, cardiography tended to reveal evidence of hypertrophy of the left ventricle although it might reveal hypertrophy of both ventricles also. Cardiography may reveal hypertrophy of the right ventricle or of both ventricles in cases in which the right ventricle is of normal size or large. These findings conform to the observations of Keith et al. (1958) and Davignon et al. (1961).

\section{SUMMARY}

This report is based on an anatomical, electrocardiographic, and clinical study of 12 cases of pulmonary atresia with an intact ventricular septum. The right ventricle was minute or small in 9 of the 12 , and it was of normal size or large in the 3 remaining cases.

Tricuspid malformations identifiable as various degrees of Ebstein's malformation are commonly observed in all cases regardless of the size of the right ventricle but among these the state of competence of the tricuspid valve appeared to vary. Enlargement of the right ventricle appears to depend on the association of significant tricuspid regurgitation.

Relief of obstruction of the pulmonary valve appears to promise beneficial results in cases with the right ventricle of normal size or large, but it seems useless in those with the right ventricle 
minute or small. In the latter group, the small size of the right ventricle limits the amount of blood that may enter the right ventricle.

Distinction of cases in which the right ventricle is minute or small from those in which it is of normal size or large depends on the radiological and cardiographic findings. Electrocardiography usually shows evidence of hypertrophy of the left ventricle in cases in which the right ventricle is minute or small, and it usually shows evidence of hypertrophy of the right ventricle or of both ventricles in cases in which the right ventricle is of normal size or large.

\section{REFERENCES}

Benton, J. W., Jr., Elliott, L. P., Adams, P., Jr., Anderson, R. C., Hong, C. Y., and Lester, R. G. (1962). Pulmonary atresia and stenosis with intact ventricular septum. Amer. J. Dis. Child., 104, 161.

Burchell, H. B., DuShane, J. W., and Brandenburg, R. O. (1960). The electrocardiogram of patients with atrioventricular cushion defects (defects of the atrioventricular canal). Amer. J. Cardiol., 6, 575.

Davignon, A. L., Greenwold, W. E., DuShane, J. W., and Edwards, J. E. (1961). Congenital pulmonary atresia with intact ventricular septum: clinicopathologic correlation of two anatomic types. Amer. Heart J., 62, 591.

Greenwold, W. E., DuShane, J. W., Burchell, H. B., Bruwer, A., and Edwards, J. E. (1956). Pulmonary atresia with intact ventricular septum. Proc. 29th Scientific Sessions, American Heart Association, p. 51.

Keith, J. D., Rowe, R. D., and Vlad, P. (1958). Heart Disease in Infancy and Childhood, p. 569. Macmillan, New York.

Kieffer, S. A., and Carey, L. (1963). Pulmonary atresia with intact ventricular septum. Brit. Heart J. In the press.

Williams, R. R., Kent, G. B., Jr., and Edwards, J. E. (1951). Anomalous cardiac blood vessel communicating with the right ventricle: observations in a case of pulmonary atresia with an intact ventricular septum. A.M.A.Arch. Path., 52, 480. 